\title{
Fast Monte Carlo algorithm for supercooled soft spheres
}

\author{
Tomás S. Grigera and Giorgio Parisi \\ Dipartimento di Fisica, Universitá di Roma La Sapienza and INFN Sezione di Roma La Sapienza, \\ Piazzale A. Moro 2, 00185 Roma, Italy
}

(January 16, 2001)

\begin{abstract}
We present a nonlocal Monte Carlo algorithm with particle swaps that greatly accelerates thermalization of soft sphere binary mixtures in the glassy region. Our first results show that thermalization of systems of hundreds of particles is achievable, and find behavior compatible with a thermodynamic glass transition.
\end{abstract}

PACS 05.10.Ln Monte Carlo methods, 64.70.Pf Glass transitions

The dynamics and thermodynamics of supercooled liquids and glasses have received much attention for some time, but are still an open problem [1]. There has been theoretical progress regarding equilibrium dynamics [2] and thermodynamics (where older ideas [3, 1 , have been recently developed [5, 6, 0,8,9, 9, 10]) as well as nonequilibrium dynamics (mainly based on analogies with spin glass behavior [11]). Many issues are open [12], such as the description of the dynamics of finite-range systems, or the existence of a thermodynamic glass transition, which is not accepted by all researchers [13].

Given the great complexity of an analytical treatment, here as in other branches of science computer simulations play a major role [14. However, simulations face difficulties, particularly in the study of equilibrium properties [14. Well-known methods such as Molecular Dynamics (MD) and traditional Monte Carlo (MC), with dynamics that closely resembles that of real systems, suffer from a slow-down at temperatures approaching the glass transition $T_{g}$. Just as a glass well below $T_{g}$ remains out of equilibrium in the laboratory, model systems require prohibitively long CPU time to equilibrate, even for a handful of particles.

Thus in recent years several algorithms have been proposed endowed with a dynamics different from real systems, one that would allow them to more easily jump across the free energy barriers that slow down MD or MC. We can mention replica MC [15], multicanonical MC [16], entropic sampling [17], parallel tempering (PT) [18], expanded ensembles MC [19], and the pivot cluster algorithm 20,21. Among these, parallel tempering is very convenient because of its straightforward implementation and ease of parallelization, and because it can be applied to both MD and MC. It has been used successfully for spin glasses for several years, and recently applied to structural glasses [22,23. However, even with these improvements, equilibration of deeply supercooled liquids requires a huge amount of $\mathrm{CPU}$ time for systems of $N \approx 50$ or more particles.

In this paper we present a Monte Carlo algorithm with a simple but nonlocal dynamics which is suited for the study of the soft sphere binary mixture, a model structural glass former widely studied in the past 24,25,26, 27.
This algorithm outperforms PT for soft spheres. It allows to equilibrate small systems with modest CPU time, and gives hope of equilibrating deeply supercooled systems of hundreds of particles.

The soft spheres binary mixture is, with appropriately chosen parameters, a fluid in which crystallization is strongly inhibited [24]. Half of the particles are of type $A$ and half of type $B$, with radii $\sigma_{A}$ and $\sigma_{B}$ respectively and unit mass. The potential energy is

$$
\mathcal{V}=\sum_{i \neq j}^{N}\left(\frac{\sigma_{i}+\sigma_{j}}{\left|\mathbf{r}_{i}-\mathbf{r}_{j}\right|}\right)^{12} .
$$

The radii are determined by fixing their ratio to be $\sigma_{B} / \sigma_{A}=1.2$ and by setting the effective diameter to unity, which for equal number of $A$ and $B$ amounts to the condition

$$
\left(2 \sigma_{A}\right)^{3}+2\left(\sigma_{a}+\sigma_{b}\right)^{3}+\left(2 \sigma_{B}\right)^{3}=4 l_{0}^{3},
$$

where $l_{0}$ is the unit of length. Under these conditions, all excess thermodynamic properties depend only on $\Gamma=\rho T^{-1 / 4}$, with $\rho=N / V$ the density and $T$ the temperature. We have chosen Boltzmann's constant $k_{B}=1$, and we restrict ourselves to the case $\rho=1$. The glass transition is known to happen at $\Gamma_{c}=1.45$ [25]. Note that with this choices, the energy and temperature are dimensionless.

We simulate this model with an algorithm that combines standard Monte Carlo moves with nonlocal moves (particle swaps) [28]. A step of the algorithm can be described as follows:

- Choose a random particle $i$.

- Draw 8 random numbers $r_{1}, \ldots, r_{8}$ uniform in $[0,1]$.

- If $r_{1}<1-p_{S}$ then

- Generate a standard MC trial move (i.e. shift particle by $\left.\Delta \mathbf{r}=\left(2 r_{2}-1,2 r_{3}-1,2 r_{4}-1\right) \Delta r\right)$

else

- Choose a random particle $j$ of a different type. 
- Swap particle positions $\mathbf{r}_{i} \leftrightarrow \mathbf{r}_{j}$.

- Shift both particles as in the standard move $\left(\Delta \mathbf{r}_{i}=\left(2 r_{2}-1,2 r_{3}-1,2 r_{4}-1\right) \Delta r, \Delta \mathbf{r}_{j}=\right.$ $\left.\left(2 r_{5}-1,2 r_{6}-1,2 r_{7}-1\right) \Delta r\right)$.

- Accept or reject new configuration according to the Metropolis criterion, i.e. accept it if $r_{8}<$ $\exp \left[-\beta\left(E_{\text {new }}-E_{\text {old }}\right)\right]$.

- Repeat for all particles.

$\Delta r$ and $p_{S}$ are the algorithm parameters. $\Delta r$ is chosen at each temperature to achieve a shift acceptance ratio around 0.5 , as is normally done in $\mathrm{MC}$ simulations. $p_{S}$ is the swap attempt probability, which we choose equal to 0.1 . Implementation of this algorithm (which we shall call swap Monte Carlo (SW) algorithm) is straightforward. Also, it should be obvious that SW satisfies detailed balance.

Note that at high densities, even the swap of two neighboring particles is a cooperative (hence slow) process. The particles must go around each other, but they are hindered by the "cage" the rest of the particles build around them. The idea behind the SW algorithm is to accelerate these processes by making them non-cooperative.

We have run SW for systems of $N=34$ and $N=800$ particles in a cubic box with periodic boundary conditions at several values of $\Gamma$ up to $\Gamma=2$, deep in the glassy region. For the larger system, a long-range cutoff at $r_{c}^{2}=3$ was imposed.

We start by comparing the approach to equilibrium of a system of $N=34$ particles simulated with standard MC, parallel tempering MC and the SW algorithm. The PT data are from ref. [22 and were obtained in a PT run with 13 replicas with $\Gamma=1,1.05, \ldots, 1.2,1.3, \ldots, 2$. The evolution of the (potential) energy per particle for each algorithm starting from a random configuration is shown in figs. 1 and 2 for temperatures above and below the transition. At $\Gamma=1.4$ (above the transition), all algorithms equilibrate rapidly. At $\Gamma=1.8$, however, it is clear that relaxation times have soared, and the three algorithms behave differently. SW is seen to relax much faster than MC and PT.

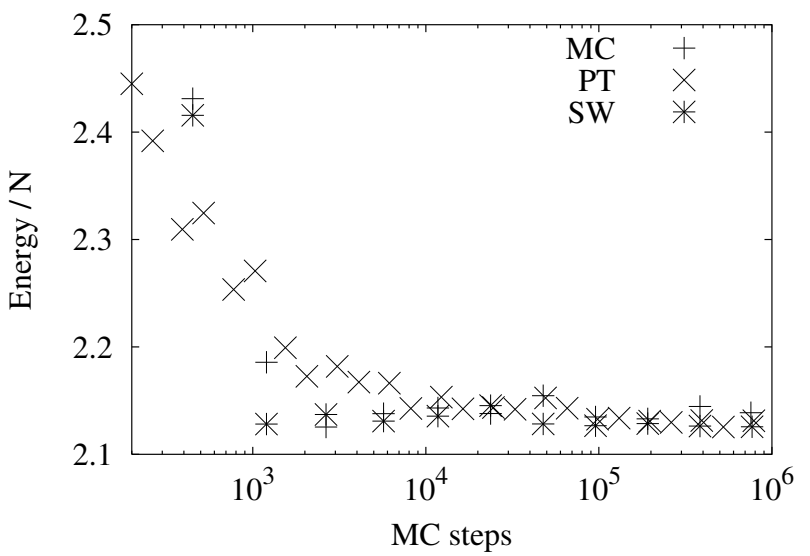

FIG. 1. Comparison of the evolution the energy starting from a random configuration for the three algorithms at $\Gamma=1.4$.

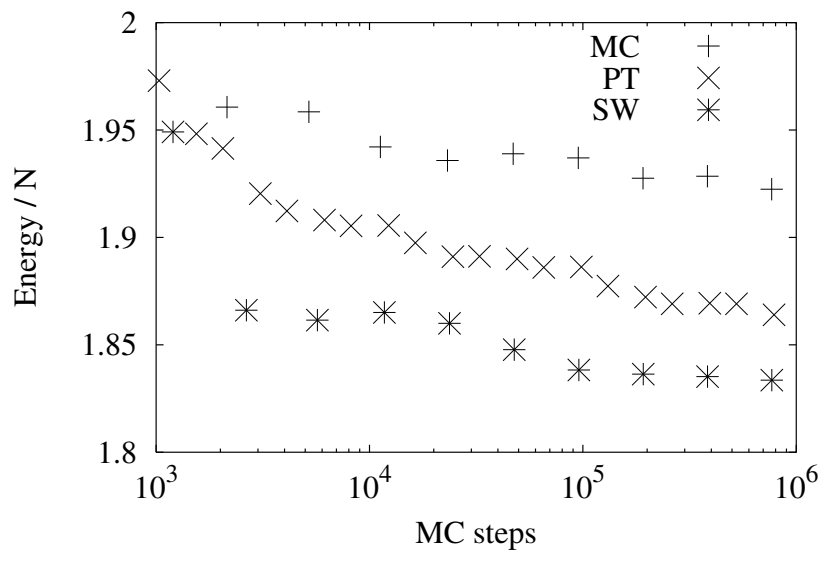

FIG. 2. As fig. 1 but for $\Gamma=1.8$.

It may seem surprising that SW works at all, i.e. that the swaps are ever accepted. But the radii of the spheres are not very different from each other, and both species are present in equal amounts, so the replacement of one species by the other is not always very unfavorable. Indeed, the swap acceptance ratio is very small (at $\Gamma=1.8$ it is about $10^{-4}$ ). However, this means that a small fraction of particles can escape the cage effect, with dramatic consequences for the dynamics.

To check whether SW is capable of equilibrating the system we run simulations with 2 different initial configurations at $\Gamma=1.4,1.5, \ldots, 2$. We find that after $1-2$ million steps (about 80 minutes of CPU time on an Alpha workstation), both configurations stabilize at the same energy. The runs are then continued up to $10^{7}$ steps, using the last $8 \cdot 10^{6}$ steps to compute the energy and specific heat. We check thermalization in two ways:

- We divide the last part of the run in blocks of 1 million steps and verify that there is no systematic shift in the average energy of these blocks.

- We compute the specific heat in two different ways, through $C_{V}=\partial\langle E\rangle / \partial T$ and through $C_{V}=\left(\left\langle E^{2}\right\rangle-\right.$ $\left.\langle E\rangle^{2}\right) / T^{2}$, and find agreement between the two.

The energy and specific heat are shown in figs. 3 and 1 as a function of temperature. The specific heat obtained from fluctuations agrees with the value from the derivative of the energy, showing that the system has thermalized. A broad but clear peak around $T \approx 0.12$ is found [29. 


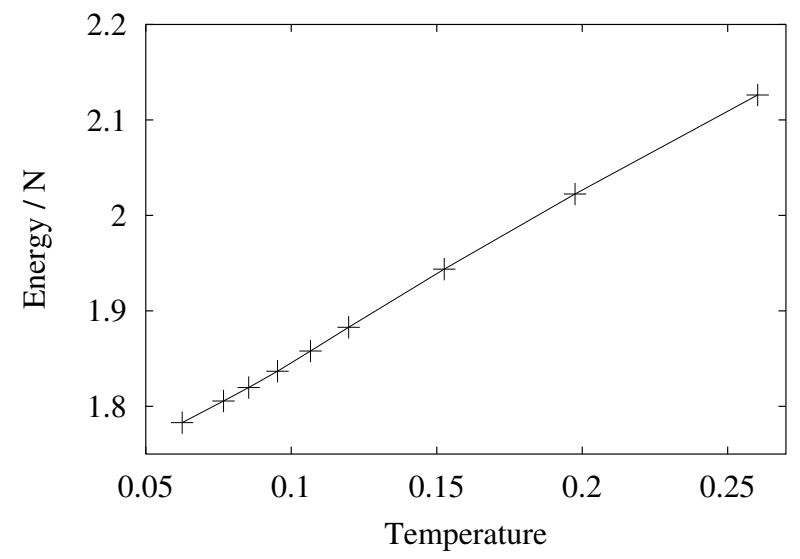

FIG. 3. Average energy vs. temperature for $N=34$. Lines are only to join neighboring points.

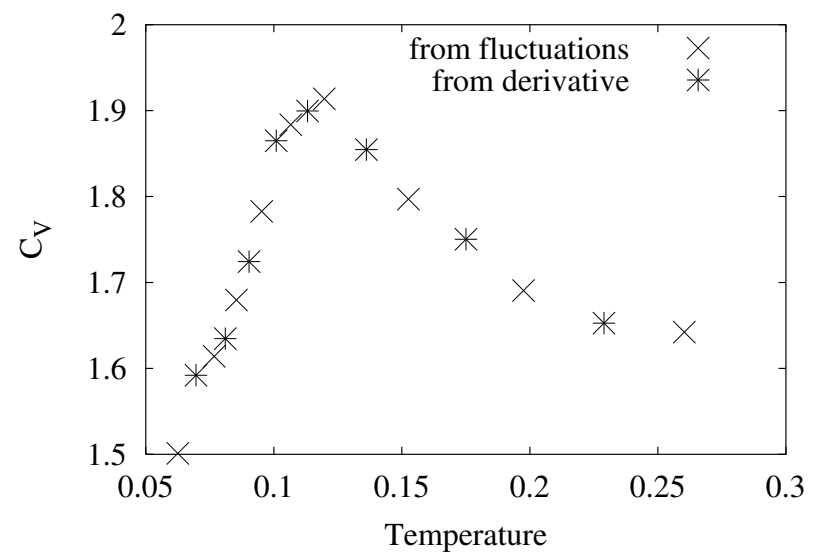

FIG. 4. Specific heat vs. temperature for $N=34$. The values obtained from the derivative of the energy and from energy fluctuations fall on the same curve.

Next we simulate a system of $N=800$ particles at

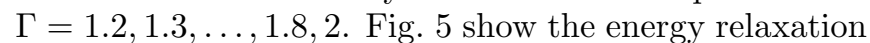
for runs of $10^{6}$ steps. The lowest temperatures clearly have not reached equilibrium in this case. The energy relaxation (after an initial fast decay which corresponds to the equilibration time for the liquid phase, namely $10^{4}$ steps) can be described by a power law $E=E_{0}+t^{-\alpha}$, with $\alpha$ strongly temperature dependent (fig. 6). A sharp change in slope of the $\alpha$ vs. $T$ curve is observed near $T \approx 0.15$. The asymptotic energy (fig. 7 ) also shows singular behavior at this temperature, as evidenced by the specific heat (fig. 8) estimated from the derivarive of the asymptotic energy.

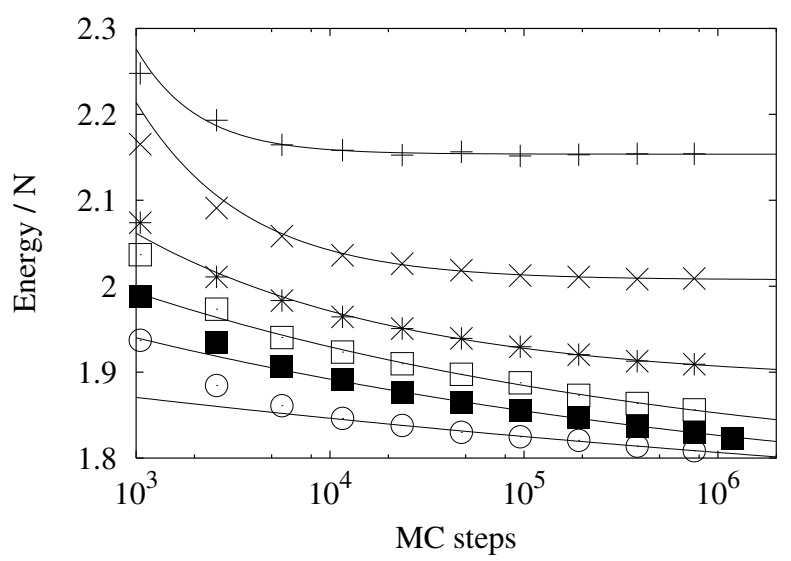

FIG. 5. Energy vs. Monte Carlo time for $N=800$, averaged over 4 initial configurations. From top to bottom, $\Gamma=1.4,1.5,1.6,1.7,1.8,2$. Lines are power law fits.

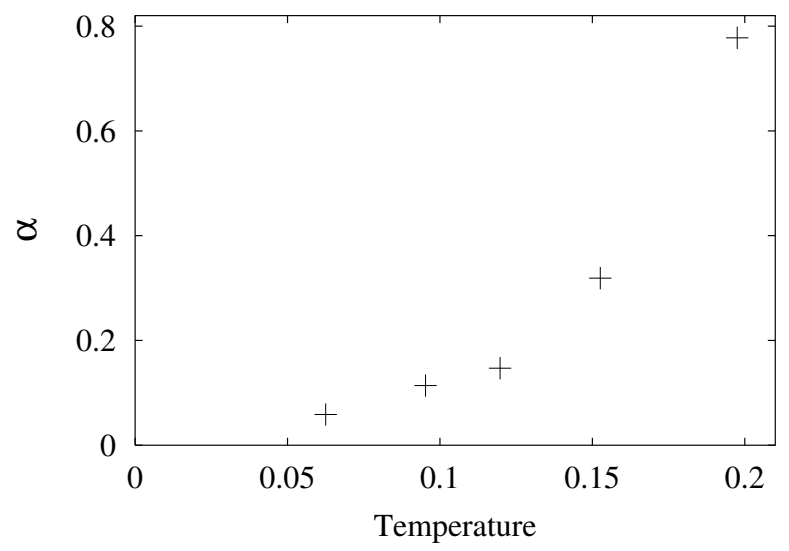

FIG. 6. Exponent $\alpha$ of the energy relaxation vs. temperature.

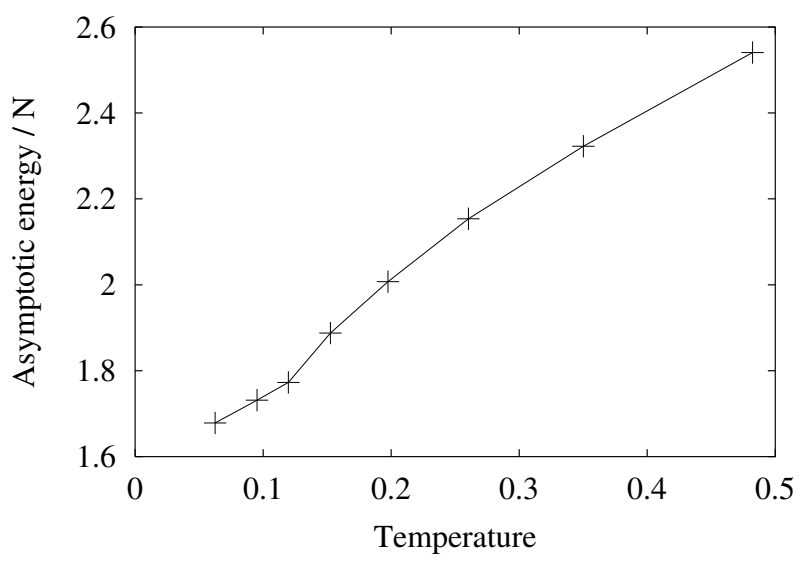

FIG. 7. Asymptotic energy vs. temperature. Lines are only to join neighboring points. 


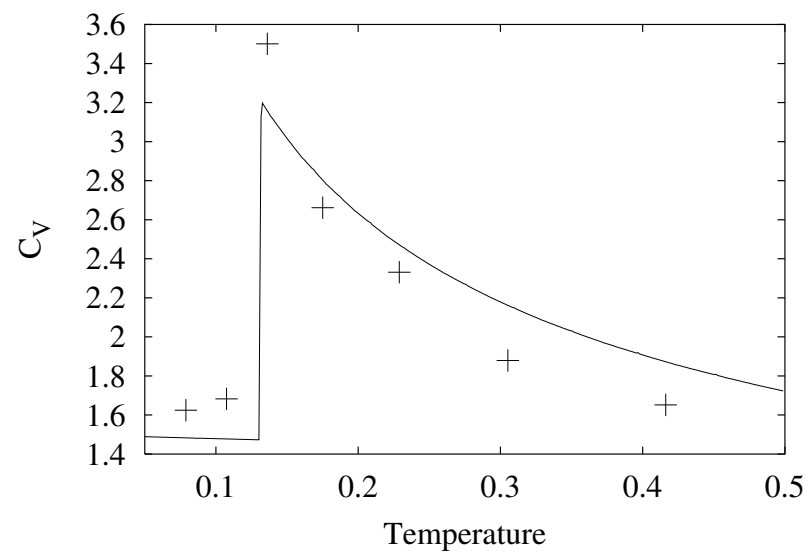

FIG. 8. Specific heat vs. temperature for $N=800$ obtained from the asymptotic value of the energy (points) with theoretical prediction from ref. [8] (continuous curve).

Equilibrium simulations at low temperatures are relevant to the question of the existence of a thermodynamic glass transition driven by an entropy crisis [3, 30]. In this respect it is to note that the peak in the specific heat of the $N=34$ system (fig. 4 ) at $T \approx 0.12$ corresponds to $\Gamma \approx 1.7$, i.e. close to the predicted [8] critical temperature $\Gamma_{K} \approx 1.65$ at which a thermodynamic transition would take place and where the calculated specific heat shows a sharp drop to a value of $3 / 2$ in the low temperature phase.

Remarkably, a similar but sharper behaviour is found for the $N=800$ system, though admittedly these values, resulting from extrapolation, should be taken with care. In figure 8 we also show the theoretical prediction for the specific heat (from [8]). There is a difference in the liquid phase, which is to be expected since the hypernetted chain approximation (HNC) used is known not to perform very well at low temperatures. But the position of the jump, at $\Gamma \approx 1.7$, is in good agreement with the theoretical $\Gamma_{K} \approx 1.65$ and with the position of the peak for the $N=34$ system. The sharp change in the slope of the dynamic exponent $\alpha$ vs. $T$ curve takes place at this same temperature.

In summary, we have presented a very efficient MC algorithm for equilibration of a soft spheres binary mixture. We have managed to equilibrate an $N=34$ system up to $\Gamma=2$ in roughly $2 \cdot 10^{6}$ steps (about 80 minutes of CPU time on an Alpha workstation). Thus the SW algorithm puts thermalization of undercooled systems of hundreds of particles within reach of modern CPUs. Our first results with SW find behavior compatible with a thermodynamic glass transition.

It would be very interesting to apply SW to other binary systems. However, it is likely that it will need some modification to achieve a reasonable swap acceptance ratio.

We thank B. Coluzzi for useful discussions. TSG is fellow of the Consejo Nacional de Investigaciones Científicas y Técnicas (CONICET, Argentina).
[1] For reviews, see C. A. Angell, Science 267, 1924 (1995) and following articles; M. D. Ediger, C. A. Angell and S. R. Nagel, J. Phys. Chem. 100, 13200 (1996).

[2] W. Götze and L. Sjögren, Rep. Prog. Phys. 55, 241 (1992).

[3] J. H. Gibbs and E. M. DiMarzio, J. Chem. Phys. 28, 373 (1958).

[4] G. Adam and J. H. Gibbs, J. Chem. Phys. 43, 139 (1965).

[5] U. Mohanty, Physica A 177, 345 (1991).

[6] F. H. Stillinger, Science 267, 1935 (1995).

[7] M. Mézard and G. Parisi, Phys. Rev. Lett. 82, 747 (1999); J. Chem. Phys. 111, 1076 (1999).

[8] B. Coluzzi, M. Mézard, G. Parisi and P. Verrocchio, J. Chem. Phys. 111, 9039 (1999).

[9] B. Coluzzi, G. Parisi and P. Verrocchio, Phys. Rev. Lett. 84, 306 (2000); J. Chem. Phys. 112, 2933 (2000).

[10] For a breif review see M. Mezard, cond-mat/0005173.

[11] J.-P. Bouchaud, L. F. Cugliandolo, J. Kurchan and M. Mézard in: Spin glasses and random fields, edited by A. P. Young, World Scientific, Singapore (1998).

[12] For a recent review of the theoretical situation in glasses see the proceedings of the workshop Unifying concepts in glass theory, Trieste (Italy) in J. Phys.: Condens. Matter 12, 6295-6682 (2000).

[13] L. Santen and W. Krauth, Nature 405, 550 (2000).

[14] W. Kob, J. Phys.: Condens. Matter 11, R85 (1999).

[15] R. H. Swendsen and J. S. Wang, Phys. Rev. Lett. 57, 2607 (1986).

[16] B. A. Berg and T. Neuhaus, Phys. Rev. Lett. 68, 9 (1992).

[17] J. Lee, Phys. Rev. Lett. 71, 211 (1993).

[18] E. Marinari and G. Parisi, Europhys. Lett. 19, 451 (1992).

[19] A. P. Lyubartsev, A. A. Martsinovsky, S. V. Shekunov and P. N. Vorontsov-Velyaminov, J. Chem. Phys. 96, 1776 (1992).

[20] C. Dress and W. Krauth, J. Phys. A: Math. Gen. 28, L597 (1995).

[21] J. G. Malherbe and S. Amokrane, Mol. Phys. 97, 677 (1999).

[22] B. Coluzzi and G. Parisi, J. Phys. A: Math. Gen. 31, 4349 (1998).

[23] R. Yamamoto and W. Kob, Phys. Rev. E 61, 5473 (2000); W. Kob, C. Brangian, T. Stühn and R. Yamamoto, condmat/0003282

[24] B. Bernu, J.-P. Hansen, Y. Hiwatari and G. Pastore, Phys. Rev. A 36, 4891 (1987).

[25] J.-L. Barrat, J.-N. Roux and J.-P. Hansen, Chem. Phys. 149, 197 (1990).

[26] J.-P. Hansen and S. Yip, Trans. Theory Stat. Phys. 24, 1149 (1995).

[27] G. Parisi, Phys. Rev. Lett. 79, 3660 (1997); Phil. Mag. B 77, 257 (1998).

[28] Though never studied in detail, algorithms with particle swaps have been used before in different problems, see D. Gazzillo and G. Pastore, Chem. Phys. Lett. 159, 388 (1989).

[29] See also recent 2D simulations by H. Fynewever and P. 
Harrowell, J. Phys.: Condens. Matter 12, 6305 (2000).

[30] W. Kauzmann, Chem. Revs. 43, 219 (1948). 